\title{
Battery Energy Storage Capacity Optimisation for Grid-Connected Microgrids with Distributed Generators
}

\author{
Rasoul Garmabdari, Mojtaba Moghimi, Fuwen Yang, Evan Gray and Junwei Lu \\ School of Engineering, Griffith University, Nathan Campus, Nathan, QLD 4111, Australia \\ Email:rasoul.garmabdari@Griffithuni.edu.au
}

\begin{abstract}
This paper presents a battery capacity optimisation method with the aim of investment and operational cost reduction for grid-connected microgrids consisting of dispatchable generators, renewable energy resources and battery energy storage. The operating cost of grid-connected commercial Microgrids is mainly associated with the purchased energy from the grid and monthly peak demand. Hence, mitigating the peak value by the means of battery energy storage and dispatchable generators during the peak period can effectively reduce the operating cost. However, due to the high cost and short life span of the battery energy storage systems, the optimum design of energy storages is of the utmost importance to the Microgrids. This paper proposes an efficient iterative method with an inner unit commitment optimisation layer to achieve the optimised battery capacity. In order to implement the inner unit commitment optimisation, the Mixed Integer Quadratic Programming (MIQP) optimisation algorithm is applied and CPLEX solver is chosen to solve the optimisation problem. This approach is applicable and beneficial when dealing with high demands as it economically distributes the load requirement between the battery and dispatchable generators. Finally, the proposed method is applied to determine the battery capacity of the experimental Microgrid at Griffith University. The simulation results for the understudy case verified the efficiency and effectiveness of the proposed approach.
\end{abstract}

Keywords-Battery Energy Storage Systems, Unit Commitment, Battery Capacity Optimisation, Grid-Connected Microgrid, Renewable Energies

\section{INTRODUCTION}

Microgrids (MG) are defined as small-scale combined energy systems to localise the electricity production by the means of various generators [1]. Microgrids are principally composed of Distributed Generators (DG), Renewable Energy Resources (RES) and loads. Battery Energy Storage Systems (BESS) have been frequently addressed as a potential candidate to solve the MGs issues such as the uncertainty and intermittency of renewable resources, wide load fluctuations, frequency and voltage instabilities [2], [3]. Addressing these issues will lead to reliability and security improvement [4], [5]. The prime function of the BESS is to absorb the excess energy being produced by RESs and return the stored energy while the system lacks energy which could occur as a result of a high demand or power drop for a short period [6]. Hence, the BESS should be able to cover the load demand in the case of power deficiency and emergency. Even though a massive BESS size provides higher flexibility and reliability, the investment cost and operational life span of the battery restrict the profit that can be achieved through the BESS. Therefore, besides the technical criteria, the economic limitations should be taken into account whilst designing the BESS for the MGs [2], [7].

In order to address the BESS optimal sizing problem in an MG, various approaches have been proposed as summarised in [8], each one of which focusing on a particular aspect of the MG leading to form different solution strategies. The optimal BESS size was achieved based on energy and power balance with an emphasis on meeting the load requirement up to a definite level of reliability in [9], whereas, in [10] the priority is to reduce the effect of RESs uncertainty at the output power and convert it to a DG. In some cases, the primary aim is to reduce the MG operational cost and therefore the BESS capacity is configured so that the maximum benefit is obtained [11]. In order to reduce the operating cost of the MG, it is essential to ascertain the optimal schedule of the generators which necessitates solving Unit Commitment (UC) optimisation problem. The UC problem involves determining the optimal configuration of DGs and scheduling BESS [4]. In [2], the optimal operational management was considered as the objective of the grid connected MG and only the price of the purchased power has been taken in to account. The authors in [3] addressed the BESS sizing problem using UC optimisation approach from the utility companies' perspective for the MG networks and subsequently considering similar criteria as in [2]. Although in [4], the UC optimisation method was proposed to determine the BESS capacity for a grid-connected MG, the peak shaving cost element has not been included in the cost function of the MG. In this paper, the UC optimisation approach is used for peak shaving and energy cost reduction to determine the BESS capacity for a grid-tied MG.

To solve the UC problem, various methods have been reported such as Dynamic Programming (DP) [12], Lagrangian Relaxation (LR), and Priority List (PL) [13]. However, increasing the number of generators would increase the number of possible solutions and the complexity of the UC problem exponentially. Later on, evolutionary algorithms were introduced to find the best generators' configuration with the aim of computational time and operating cost reduction as explained in [14] and [13]. In this manuscript, the Mixed Integer Quadratic Programming (MIQP) approach is used to solve the UC optimisation problem.

In this paper, it is aimed to achieve the optimum BESS capacity for an MG including DGs and RESs through the operational scheduling of generators and BESS of the MG. The rest of the paper is as follows. In section II, the contributing 
components to the operating cost of the $\mathrm{MG}$ are explained and then, the objective function and solution strategy are constituted accordingly. The cost involved with the BESS is expressed in this section. In section III, the developed solution is applied to a case study to verify the effectiveness of the proposed method. The achieved optimised BESS, as well as MG operation over the scheduling horizon, are discussed in the result and analysis section. Finally, the last section draws some conclusions based on achieved results.

\section{THE MICROGRID CONFIGURATION AND PROBLEM FORMULATION}

A grid-connected MG typically consists of RESs, DGs, and BESS to meet the load requirement as illustrated in Fig. 1. The quantity and types of the DGs can be varied depending on the load characteristics, the availability of the fuel, the budget limit and other factors [15]. The RES represented in Fig. 1 encompasses solar photovoltaic panels (PV) and Wind Turbine (WT).

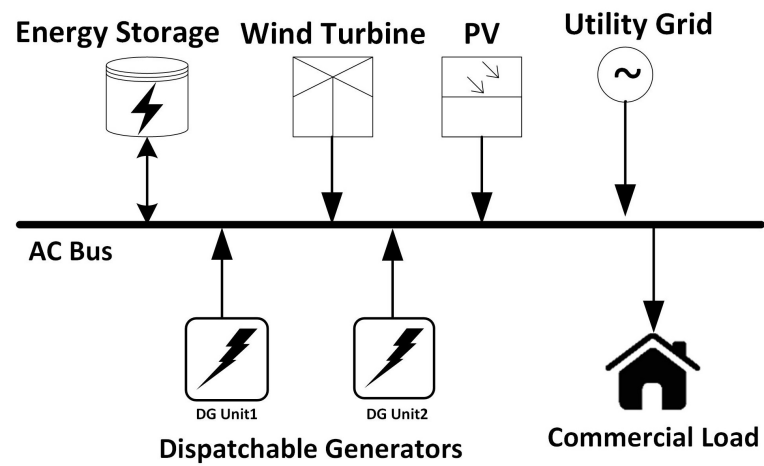

Figure 1. General architecture of a commercial microgrid.

The DGs should be supplied by fuel to operate whereas WT and PV are cost free energy sources. The BESS is utilised to store the excess RESs generated energy and deliver this energy during high demand time. The BESS, RESs, DGs, and utility grid are to fulfil the commercial load requirement through $\mathrm{AC}$ Bus at all time.

\section{A. Unit Commitment Optimisation Problem}

For the purpose of BESS sizing, it is essential to identify the contributing elements to the BESS cost and the technical criteria and constraints of the MG that should be satisfied.

In the MG, each generator unit has different operational cost and hence, their configuration at each time step determines the system operational cost. The sum of the operational cost within a certain period results in the System Scheduling Cost (SSC) which should be minimised while specifying the BESS capacity for the MG. To minimise the SSC, the UC optimisation problem should be solved for the MG. This approach guarantees the superior configurations at each time instant $t$, in which the minimum operating cost is imposed.

The SSC optimisation problem is solved for a certain short period of time. In this paper, the operation period is considered to be 24 hours. The expenses involved with the SSC optimisation problem encompasses mainly the fuel cost of DGs, the shutdown and start up costs, the BESS operational cost, and the cost of power exchange with the grid. The fuel cost function for every individual unit differs from other units and fuel consumption at each time instant depends on the output power of the unit at that time. The fuel cost for DGs is commonly modelled as a quadratic function as in (1).

$$
F(P(t), j)=\alpha_{j} \cdot P_{j}^{2}(t)+\beta_{j} \cdot P_{j}(t)+\gamma_{j}
$$

where the $P_{j}(t)$ is the output power of the $j^{t h}$ unit at time step t. $\alpha, \beta$, and $\gamma$ are cost coefficients for a particular unit.

In order to calculate the SSC as stated in (2), the transition status and the fuel cost of DGs are taken into account at each time step as starting or shouting down the units will be costly. The transition cost can be varied for each individual unit according to its type and technical specifications. Another component associated with the SSC is the exchanged power between MG and the utility grid that should be included in calculation as formulated in (11). The status of the previous time instant for each unit is investigated to decide whether to charge the start up or shutdown cost at each time step.

$$
\begin{gathered}
S S C=\sum_{t=1}^{T}\left(\sum _ { j = 1 } ^ { N } \left(F(P(t), j) \cdot U_{j}(t)+S t_{j} \cdot\left(1-U_{j}(t-1)\right)\right.\right. \\
\left.U_{j}(t)+S d_{j} \cdot\left(1-U_{j}(t)\right) \cdot U_{j}(t-1)\right)+T E C(t)+ \\
\left.C_{o p} \cdot P_{B E S S}(t)\right)
\end{gathered}
$$

where $\mathrm{T}, \mathrm{N}, U_{j}(t)$, and $T E C(t)$ denote the operating time horizon, the total number of DGs, the operational status of the DG $j$ and the total electricity cost exchanged with the utility grid at time $t$, respectively. The start up and shutdown cost of the $j^{t h}$ unit at time step $t$, the BESS power at time $t$, and the operational cost of the BESS are represented by $S t_{j}$ and $S d_{j}$, $P_{B E S S}(t)$, and $C_{o p}$, respectively.

The $P_{B E S S}(t)$ is defined as a function of discharging power $\left(P_{d i s}(t)\right)$ and charging power $\left(P_{c h}(t)\right)$ of the BESS as in (3).

$$
P_{B E S S}(t)=P_{d i s}(t)-P_{c h}(t)
$$

The aforementioned objective function is subjected to the following constraints. First and foremost, according to the energy conservation law, the sum of the generated power should be equal to the consumed power at each time instant as stated in (4).

$$
\begin{gathered}
\sum_{j=1}^{N}\left(P_{j}(t) \cdot U(t)\right)+P_{P V}(t)+P_{W T}(t)+P_{B E S S}(t) \\
+P_{\text {grid }}(t)=P_{\text {load }}(t) \\
t \in\{1,2, \ldots, T\} \quad \& \quad j \in\{1,2, \ldots, N\}
\end{gathered}
$$

where $P_{P V}(t), P_{W T}(t), P_{\text {load }}(t)$, and $P_{\text {grid }}(t)$ imply instantaneous amount of PV output power, WT output power, load, and the purchasing power from the grid, respectively.

Spinning Reserve $(\mathrm{R}(\mathrm{t}))$ is defined as the maximum available synchronised power of all DGs plus the available power in the BESS $\left(P_{B E S S}^{\max }(t)\right)$ that can be delivered to the load at 
each time slice as in (5). The $\mathrm{R}(\mathrm{t})$ is commonly considered as a fraction of the load at each time step.

$$
\begin{aligned}
\sum_{j=1}^{N}\left(P_{j}^{\max } \cdot U_{j}(t)\right) & +P_{B E S S}^{\max }(t)+\left(P_{\text {grid }}^{\max }(t)-P_{\text {grid }}(t)\right) \\
& \geq P_{\text {load }}(t)+R(t) \\
& \& \quad t \in\{1,2, \ldots, T\}
\end{aligned}
$$

Power generation and ramp rate constraints for each unit should be taken into consideration as stated in (6) and (7).

$$
\begin{gathered}
P_{j}^{\text {min }} . U_{j}(t) \leq P_{j}(t) \leq P_{j}^{\max } . U_{j}(t) \\
t \in\{1,2, \ldots, T\} \quad \& \quad j \in\{1,2, \ldots, N\} \\
R_{j}^{\text {down }} \leq P_{j}(t)-P_{j}(t-1) \leq R_{j}^{u p}
\end{gathered}
$$

where $P_{j}^{\min }$ and $P_{j}^{\max }$ denote the minimum and maximum output power of the unit j. $P_{j}(t-1), R_{j}^{\text {down }}, R_{j}^{u p}$ are the output power of unit $\mathrm{j}$ at previous time step, the ramp down and ramp up rates of unit $\mathrm{j}$, respectively. The ramp rates are in $\mathrm{kW} /$ minute.

The charging and discharging power rates, as well as the BESS energy, are limited as in (8).

$$
\begin{gathered}
0 \leq P_{c h}(t) \leq P_{c h}^{\max } \cdot B(t) \\
0 \leq P_{\text {dis }}(t) \leq P_{d i s}^{\max } \cdot(B(t)-1) \\
E_{B E S S, \text { Min }} \leq E_{B E S S}(t) \leq E_{B E S S, \text { Max }}
\end{gathered}
$$

where $E_{B E S S}(t), P_{c h}^{\max }, P_{\text {dis }}^{\max }, E_{B E S S, \text { Min }}, E_{B E S S, M a x}$ are the BESS energy at time t, maximum BESS charging power rate, maximum BESS discharging power rate, minimum energy that must remain in the BESS all the time, and the maximum energy that the BESS can store, respectively. $\mathrm{B}(\mathrm{t})$ is the binary variable which decides whether the BESS is charging or discharging [16]. The initial and ultimate energy of the BESS should be equal within a complete cycle [17], [4].

$$
E_{\text {initial }}=E_{T}
$$

The energy exchange model of the BESS as represented in (10) determines the available energy at each time instant. This model is a function of charging and discharging rates, time $(\mathrm{t})$, the available energy at the previous time step $E_{B E S S}(t-1)$, charging $\left(\eta_{c h}\right)$ and discharging $\left(\eta_{\text {dis }}\right)$ coefficients.

$$
\begin{gathered}
E_{B E S S}(t)=E_{B E S S}(t-1) \cdot\left(1-\delta_{B E S S}\right) \\
+\left(P_{c h}(t) \cdot \eta_{c h}-\frac{P_{d i s}(t)}{\eta_{d i s}}\right) \cdot \Delta t
\end{gathered}
$$

where $\delta_{B E S S}$ and $\Delta t$ represent the energy loss ratio of the BESS and time interval. In this paper, the $\Delta t$ is considered equal to 1 hour over the study period.

As the introduced UC optimisation problem contains quadratic terms as well as integer and continuous variables, the Mixed Integer Quadratic Programming (MIQP) solvers with CPLEX optimiser in MATLAB platform has been employed to minimise the SSC.

\section{B. Proposed BESS Sizing Method}

In order to determine the BESS capacity at which the minimum cost is imposed to the MG, the proposed UC optimisation method is iteratively performed for the MG for various BESS capacities. The flow chart of the proposed algorithm is depicted in Fig. 2.

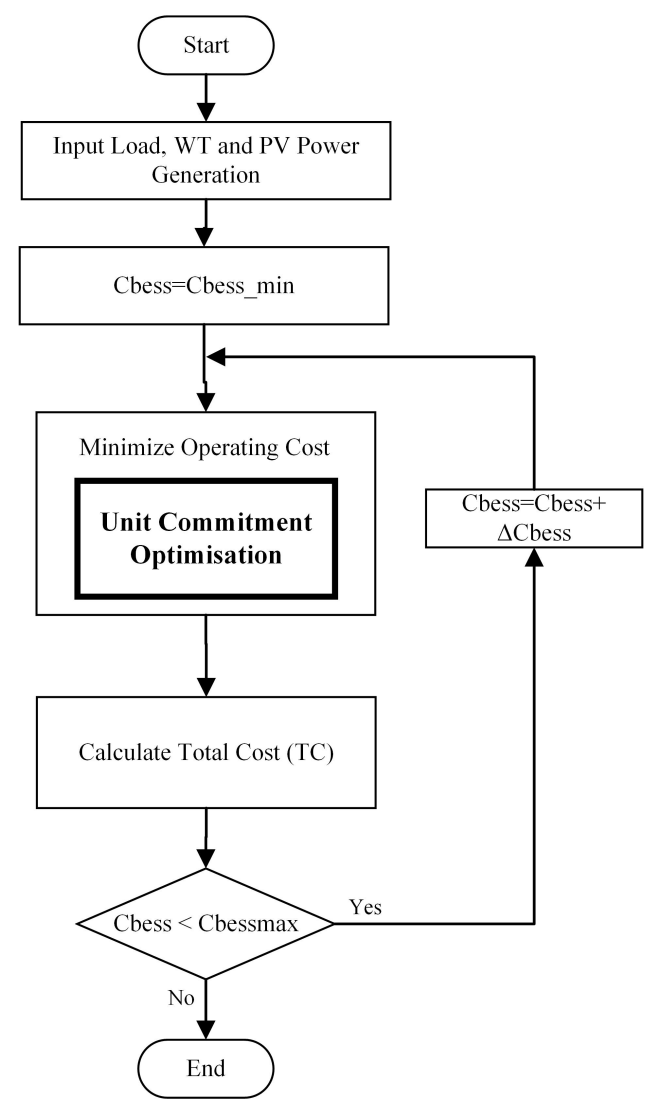

Figure 2. Flowchart of the algorithm to achieve the optimal size of the BESS.

At the first stage, the input variables including WT instant generated power, PV instant power, and the load profile are set. Then, the BESS is set to its minimum amount. Afterwards, the minimum cost and optimum scheduling of the $\mathrm{MG}$ is obtained over the operating period using the developed optimisation model in the previous section. In the next step, for the purpose of cost evaluation of the MG, the expenses associated with the BESS as well as the operational cost of the MG which is influenced by applying the BESS should be taken into account. This variable is represented by Total Cost (TC) and can be calculated through (2), (12), and (13).

At each iteration, the BESS capacity range is inspected and if it is less than its maximum value, it will be incremented by $\Delta C_{\text {bess }}$ and the operating cost minimisation stage is repeated again till the BESS capacity reaches to its maximum amount. It is noteworthy that the proposed algorithm is to be executed over a certain period of time. In this paper, the optimisation time is considered to be 24 hours. The TC varies as the size of BESS changes. Therefore, the optimum size of the BESS can be concluded at the point where the TC is minimum. In (2), the total electricity cost $(T E C)$ is calculated according to the 
total energy exchanged with the grid plus the monthly peak power as formulated in (11). Although the greatest demand is only accounted for the entire month, the price is far more expensive as compared to the energy cost $(E C(t))$. Hence, it extensively contributes to the TEC.

$$
T E C=E C(t) \cdot P_{\text {grid }}(t)+P C \cdot P_{\text {load }, \text { Peak }}
$$

where PC is the price of the peak for a month $\$ / \mathrm{kW}$ and $P_{\text {load,Peak }}$ is the peak load of the month.

As the cost and the profit made by the BESS are associated with its capacity, it is assumed that other components and their size have already been specified. The BESS cost includes capital cost $(C C)$ and operational cost, both of which are proportional to the BESS size $\left(C_{B E S S}\right)$ [3], [4]. The capital cost consists of installation and purchase costs, and the operational cost covers the maintenance cost $\left(C_{M}\right)$ during the life span (L) of the BESS. In this cost model, the annual interest rate $(r)$ has been taken into account. Finally, the total cost of BESS per day (TCPD) can be achieved using (12).

$$
\begin{aligned}
C C & =\frac{r \cdot(1+r)^{L}}{(1+r)^{L}-1} \\
T C P D & =\frac{1}{365}(C C+C M) \cdot C_{B E S S}
\end{aligned}
$$

The TC consists of two main elements comprising SSC and TCPD as expressed in (13). The SSC reduces by increasing the BESS capacity, whereas the TCPD increases linearly. The SSC variation versus BESS size is dominant up to a certain point with respect to the TCPD increase. Thereafter, the TCPD will become more significant than the financial impact of the BESS on the SSC an therefore by increasing the BESS size, the TC increases subsequently. The capacity at which the minimum TC is yielded will be the optimised size of the BESS for the MG.

$$
T C=S S C+T C P D
$$

\section{RESUlTS AND ANALYSIS}

The $T E C$ calculation in (11) reveals that reducing the peak value lessens the bill remarkably. Mitigating the peak by the BESS is a common practice being used in MGs and obviously the higher peak for a longer period, the larger BESS capacity is required. However, if the difference between the monthly peak and average value was massive, then increasing the BESS capacity to cover the monthly peak would be quite unreasonable as they are costly components with regard to their short life period as calculated in (12).

These reasons have become the motivation to introduce a technique with the help of DGs in conjunction with the BESS to reduce the cost of the $M G$ while satisfying the technical criteria. In this section, the MG components, their technical features, and the characteristics of the understudy commercial load profile are explained. In the second part, the operation of the DG's and the variation of BESS energy after applying the SSC optimisation technique are examined in details. Afterwards, the expenses and benefits related to the BESS are analysed and the procedure to determine the BESS capacity is discussed. Finally, the performance of the
MG, its financial benefit and its effect on the load profile will be scrutinised.

\section{A. Case Study}

In this study, it is aimed to specify the BESS capacity for a grid-connected MG which is expected to meet the load demand of three commercial buildings at Griffith UniversityNathan Campus (N44, N74 and N79). The MG comprises the following components: $4 \times 5 \mathrm{~kW}$ WT with a total generation capacity of $20 \mathrm{~kW}$ at their rated wind speed, $94 \times 320 \mathrm{~W}$ polycrystalline PV panels with a maximum power generation of $30 \mathrm{~kW}$, a single unit of Microturbine (MT) manufactured by Capstone Cooperation with a power range of 0 to $30 \mathrm{~kW}$ with a start up time of 15 minutes and a $30 \mathrm{~kW}$ diesel generator. The capital cost for the proposed Lithium-ion type BESS, the annual maintenance cost and interest rate are assumed to be $\$ 700$ per $\mathrm{kWh}, \$ 20$ per $\mathrm{kWh}$ and 5\%, respectively. The BESS technical specifications are tabulated in Table I.

TABLE I. THE BESS PARAMETERS

\begin{tabular}{|c|c|c|}
\hline Description & Value & Unit \\
\hline Charging Rate & 20 & $\mathrm{~kW}$ \\
\hline Discharging Rate & 10 & $\mathrm{~kW}$ \\
\hline Life Time & 3 & Years \\
\hline Charging Efficiency $\left(\eta_{c}\right)$ & 95 & $\%$ \\
\hline Discharging Efficiency $\left(\eta_{d}\right)$ & 95 & $\%$ \\
\hline Depth of Discharge $(\mathrm{DOD})$ & 10 & $\%$ \\
\hline
\end{tabular}

The load profiles of the aforementioned buildings were collected through the central data acquisition system of Griffith University. It should be mentioned that the building N79 is still being constructed, but due to its close similarity with G39 located in Gold Coast Campus with respect to the building application and architecture, the load profile of G39 was used in the calculation, instead. The total load for the day on which the peak demand has been observed is illustrated in Fig. 3. As it is clear from Fig. 3, the demand reaches to $340 \mathrm{~kW}$ at peak hour and minimum demand has been observed between the time 3 am to 7 am with the power of $223 \mathrm{~kW}$ and the average power is about $270 \mathrm{~kW}$.

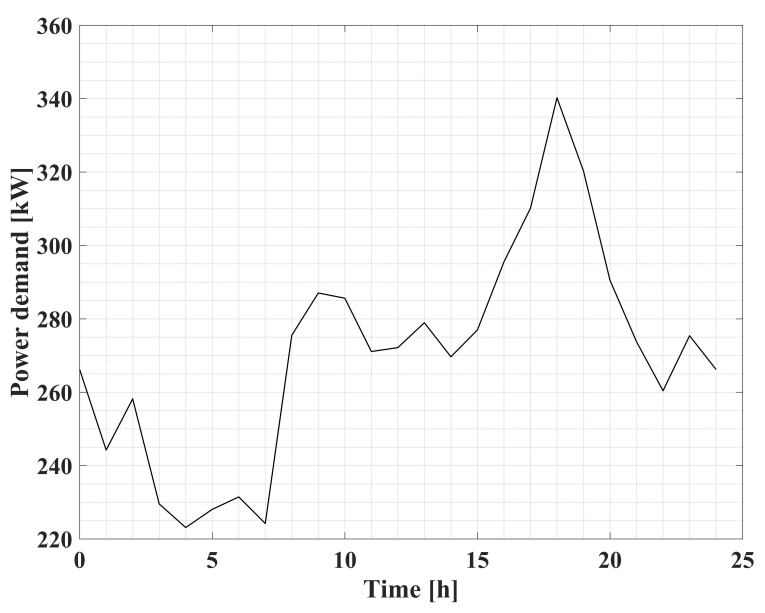

Figure 3. The total load profile of the day with the highest demand for the buildings N44, N74 and G39.

According to the Time of Use (TOU) pricing policy for commercial units in Queensland, customers are charged for 
the energy purchased from the grid and the highest monthly peak according to the prices stated in Table II.

TABLE II. THE ELECTRICITY TARIFF OF COMMERCIAL LOADS

\begin{tabular}{|c|c|c|c|}
\hline Description & Period & Price & Unit \\
\hline \multirow{2}{*}{ Purchased Energy Price } & 7 AM to 8 PM & 9.7 & $c / k W h$ \\
\cline { 2 - 4 } & 8PM to 7 AM & 6.6 & $c / k W h$ \\
\hline Peak Price & Monthly & 24 & $\$ / k W$ \\
\hline
\end{tabular}

\section{B. Unit Commitment Results}

The proposed method was employed to determine the optimum BESS capacity for the above MG and load profile. In this scenario, the BESS in conjunction with DGs are supposed to reduce the cost by operating during the peak period. As it is obvious from the load profile shown in Fig. 3, during the peak demand which falls between $3 \mathrm{pm}$ and $8 \mathrm{pm}$, the BESS is expected to be intensively discharging to compensate the power shortage. During this time which the peak value is far greater than the average demand, the deficit power is distributed between diesel generator and MT as well as the BESS.

As explained earlier, using the DGs could be more economical and efficient in the case of peak shaving, otherwise, they would not be cost worthy unless it is desired to utilise them as the backup generators. The operating status and output power of the DGs are practically related to the BESS energy at the time of the peak occurrence as well as the duration of the peak demand. As it is shown in Fig.4, the generators are run based on their operational cost during high demand period. In the occasion of this scenario, the DGs inject energy to the load for approximately $70 \mathrm{kWh}$ over the peak time. The MT is initially run to provide $10 \mathrm{~kW}$ power to the load and as the demand climbs up, the output power of MT is risen up till it reaches to its maximum power $(30 \mathrm{~kW})$. Since the demand is still increasing, the diesel generator is activated to compensate the rest of the power deficiency.

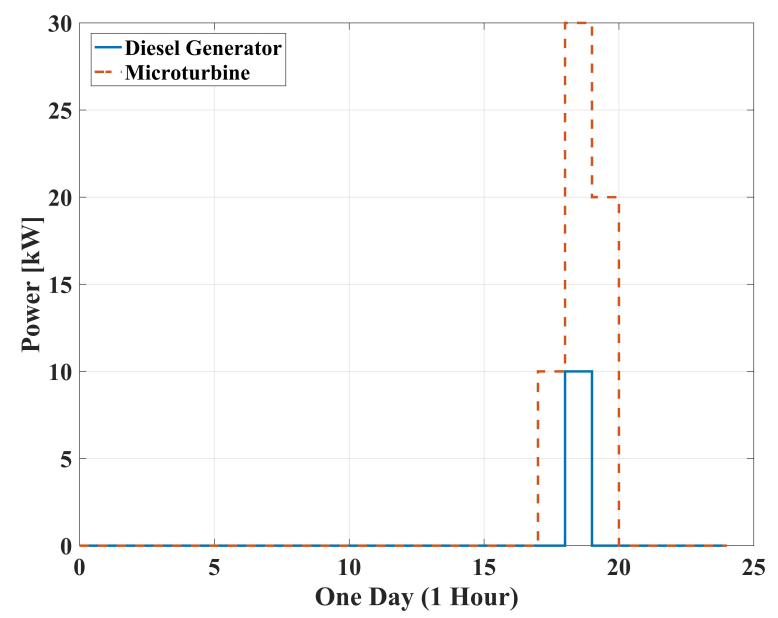

Figure 4. The operating status and generated power by MT and diesel generator over the operating period.

In the proposed method, the BESS serves as a DG and therefore it treats similar to the DG, but with regard to its constraints. The energy variations of the BESS at its optimum capacity is depicted in Fig. 5.

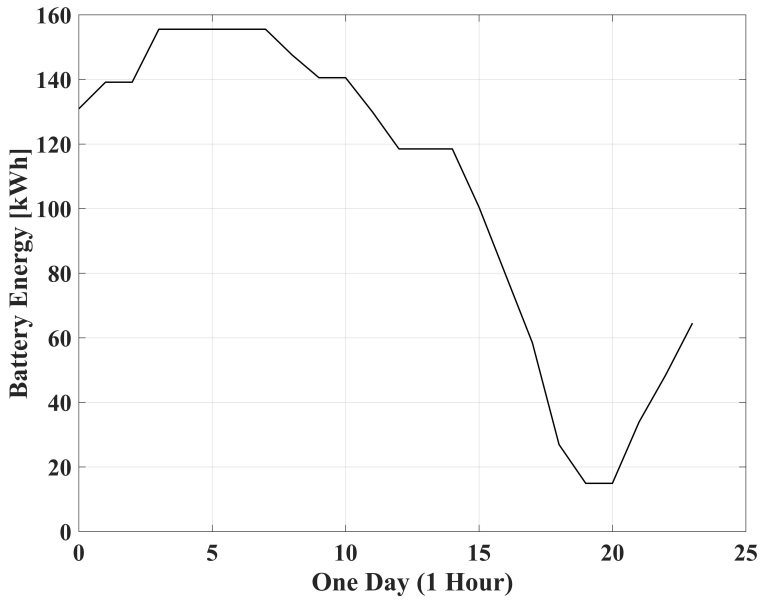

Figure 5. The BESS energy variations over the course of the day.

According to the underlying concept of this method, if the difference between the peak value and the typical variation range is massive, the scheduling objective function discussed in (2) seeks for the optimum configuration of DGs and BESS in which the total cost is minimised. The BESS is charged up to $158 \mathrm{kWh}$ during the off peak time and discharged during the high demand period between $3 \mathrm{pm}$ to $8 \mathrm{pm}$ reaching to the depth of discharge (DOD) value at $16 \mathrm{kWh}$. In other words, the UC optimisation gives higher priority to peak demands than the purchasing energy price and if no high demand is observed, the energy price will be the decisive factor in charging and discharging status of the BESS.

\section{Battery Sizing and Cost Analysis}

Considering the operating cost of DGs and the BESS cost, the proposed algorithm applies different BESS capacities and calculates the minimum attainable cost of the MG to yield the optimum BESS capacity. As the SSC is optimised through the inner optimisation layer at each iteration of the algorithm, the TC is calculated according to the specified BESS capacity. Fig.6 illustrates the TC variations as defined in (13) for various BESS configurations.

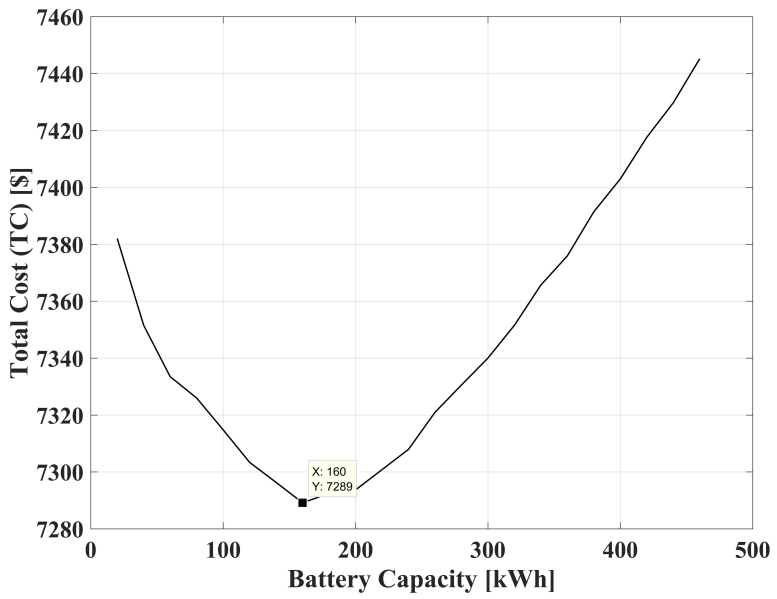

Figure 6. Total scheduling cost of the MG versus BESS capacity. 
According to Fig. 6, the optimal size of the BESS is equal to $160 \mathrm{kWh}$ as the TC is minimum $(\$ 7267)$. As it is clear from Fig. 6, the TC initially starts decreasing by increasing the BESS size. However, it is not always the case as the TC ascends after a certain BESS capacity at which the TCPD cost outweighs the profits that BESS brings to the MG.

In order to represent the effectiveness and efficiency of the proposed technique, the purchasing power from the grid after applying the method and the original demand are compared in Fig. 7. As it is conspicuous, the WT and PV are generating power all the time and they reached to their maximum power generation between 10 am to $3 \mathrm{pm}$. As the SSC objective function is to schedule the operation of the BESS and DGs, these units only contribute to the load when it is economically profitable for the MG. This strategy has led to alleviation of the purchased power from the grid throughout the period. As shown in Fig. 7, the peak power is reduced from $340 \mathrm{~kW}$ to $272.4 \mathrm{~kW}$ and subsequently the benefit resulted from peak reduction is obtained to be $\$ 1622.4$.

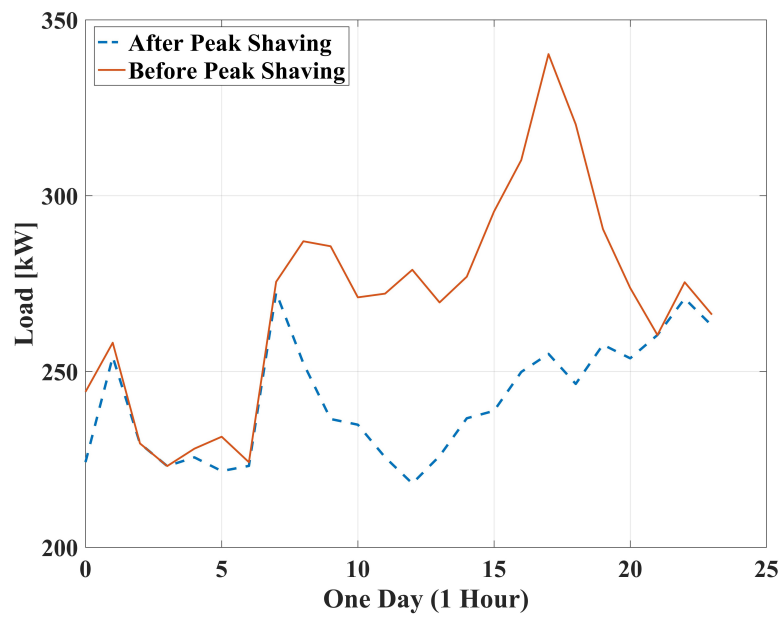

Figure 7. The peak shaved load profile.

\section{CONCLUSION}

The optimal capacity of the BESS for a grid-tied MG comprising of DGs and RESs is determined by applying a twolayer optimisation method. The generic optimisation model of the system was initially developed and then applied to the MG at Griffith University. In order to achieve the optimal size of the BESS, the operating cost, the cost of the BESS and also the cost of DGs were taken into consideration. The understudy MG consists of WT and PV as renewable generators, MT and diesel generator as DGs. Applying the proposed two layer optimisation technique to the MG, a BESS unit with $160 \mathrm{kWh}$ capacity was achieved. The operation of the BESS and generators were optimised via the developed inner UC optimisation layer such that the designed $\mathrm{MG}$ reduces the power variation range over the entire study period. The DGs and BESS participate to satisfy the load when it is economically beneficial to the MG. Consequently, the total cost of the MG is minimised by applying this strategy. The cost and performance analysis of the MG verified the effectiveness and efficiency of the represented method.

\section{REFERENCES}

[1] L. N. An, T. Quoc-Tuan, B. Seddik, and N. Van-Linh, "Optimal sizing of a grid-connected microgrid," in Industrial Technology (ICIT), 2015 IEEE International Conference on. IEEE, 2015, pp. 2869-2874.

[2] H. Xiao, W. Pei, Y. Yang, and L. Kong, "Sizing of battery energy storage for micro-grid considering optimal operation management," in Power System Technology (POWERCON), 2014 International Conference on. IEEE, 2014, pp. 3162-3169.

[3] S. Chen, H. B. Gooi, and M. Wang, "Sizing of energy storage for microgrids," IEEE Transactions on Smart Grid, vol. 3, no. 1, pp. 142151, 2012.

[4] J. P. Fossati, A. Galarza, A. Martín-Villate, and L. Fontán, "A method for optimal sizing energy storage systems for microgrids," Renewable Energy, vol. 77, pp. 539-549, 2015.

[5] C. Chen, S. Duan, T. Cai, B. Liu, and G. Hu, "Smart energy management system for optimal microgrid economic operation," IET renewable power generation, vol. 5, no. 3, pp. 258-267, 2011.

[6] J. P. Barton and D. G. Infield, "Energy storage and its use with intermittent renewable energy," IEEE transactions on energy conversion, vol. 19, no. 2, pp. 441-448, 2004.

[7] R. Dufo-López and J. L. Bernal-Agustín, "Multi-objective design of pv-wind-diesel-hydrogen-battery systems," Renewable energy, vol. 33, no. 12, pp. 2559-2572, 2008.

[8] S. Sharma, S. Bhattacharjee, and A. Bhattacharya, "Operation cost minimization of a micro-grid using quasi-oppositional swine influenza model based optimization with quarantine," Ain Shams Engineering Journal, 2015.

[9] Z. Lan, Y. Zheng, Y. Xiu, F. Yang, and C. Jie, "Optimal configuration of battery capacity in microgrid composed of wind power and photovoltaic generation with energy storage," Power System Technology, vol. 36, no. 12, pp. 26-31, 2012.

[10] J. Mitra and M. R. Vallem, "Determination of storage required to meet reliability guarantees on island-capable microgrids with intermittent sources," IEEE Transactions on Power Systems, vol. 27, no. 4, pp. 2360-2367, 2012.

[11] J. Zhang, J. Zhang, and J. Du, "Optimal battery capacity configuration for micro-grid economic planning," in Electricity Distribution (CICED), 2014 China International Conference on. IEEE, 2014, pp. 1266-1269.

[12] A. Oudalov, R. Cherkaoui, and A. Beguin, "Sizing and optimal operation of battery energy storage system for peak shaving application," in Power Tech, 2007 IEEE Lausanne. IEEE, 2007, pp. 621-625.

[13] S. Tiwari, B. Dwivedi, and M. Dave, "A two stage solution methodology for deterministic unit commitment problem," in Electrical, Computer and Electronics Engineering (UPCON), 2016 IEEE Uttar Pradesh Section International Conference on. IEEE, 2016, pp. 317-322.

[14] G. Basso, N. Gaud, F. Gechter, V. Hilaire, and F. Lauri, "A framework for qualifying and evaluating smart grids approaches: focus on multiagent technologies," Smart Grid and Renewable Energy, vol. 4, no. 04, p. 333, 2013.

[15] R. Al Abri, E. F. El-Saadany, and Y. M. Atwa, "Optimal placement and sizing method to improve the voltage stability margin in a distribution system using distributed generation," IEEE transactions on power systems, vol. 28, no. 1, pp. 326-334, 2013.

[16] A. S. Al Busaidi, H. A. Kazem, A. H. Al-Badi, and M. F. Khan, "A review of optimum sizing of hybrid pv-wind renewable energy systems in oman," Renewable and Sustainable Energy Reviews, vol. 53, pp. 185193, 2016.

[17] S. Ashok, "Optimised model for community-based hybrid energy system," Renewable energy, vol. 32, no. 7, pp. 1155-1164, 2007. 